\title{
Promoviendo la Justicia Social y la Democracia en la Formación del Profesorado: Preparación del \\ Profesorado 1.0, 2.0 y 3.0
}

\author{
Advancing Social Justice and Democracy in Teacher Education: \\ Teacher Preparation 1.0, 2.0 and 3.0
}

\author{
Promovendo a Justiça Social e a Democracia na Formação do \\ Professor: Preparação do Professor 1.0, 2.0 e 3.0
}

Kenneth Zeichner *

Universidad de Washington

\begin{abstract}
Mantener una mirada crítica hacia los programas de formación del profesorado es fundamental, tanto para pensar sobre su pertinencia como para avanzar en el desarrollo de estos programas hacia una formación que aporte y que a la vez se desarrolle en un marco de justicia social y equidad. Sin embargo, aunque muchos de los programas de formación de profesorado propugnan la justicia social, sus estudiantes no son formados en un ambiente propio de esta consigna. En Estados Unidos, el debate actual se sostiene en torno a cuál es la mejor visión: si los programas que se basan en las prácticas de campo (1.0) o aquellos que se enfocan en aspectos técnicos de la formación, así como en obtener resultados mayores en pruebas estandarizadas (2.0). Lo cierto es que ninguna de estas visiones aviva en sus prácticas valores imprescindibles e inherentes a una formación en justicia social, por lo que se hace explícita la necesidad de transformación de los programas de formación de profesorado.
\end{abstract}

Descriptores: Justicia social, Equidad, Pedagogía, Programas de formación de profesorado, Sensibilidad cultural.

\begin{abstract}
Maintaining a critical eye on the teacher education programs is essential, both to think about their relevance and to advance the development of these programs towards training that contributes to social justice, at the same time that develop within a social justice and equity framework. However, although many of the teacher education programs espouse social justice, his students are not trained in an own atmosphere of this slogan. In the United States, the current debate builds on what is the best vision if the programs that are based on field practices (1.0) or those that focus on technical aspects of training, as well as greater results on standardized tests (2.0). The truth is that none of these visions revives in their practices, essential and inherent values to training in social justice. Therefore, this article makes explicit the need for transformation of teacher education programs.
\end{abstract}

Keywords: Social justice, Equity, Pedagogy, Teacher education programs, Cultural sensitivity.

*Contacto: kenzeich@uw.edu

ISSN: 2254-3139

www.rinace.net/riejs/

revistas.uam.es/riejs
Recibido: $\quad 15$ de septiembre 2016

$1^{\text {a }}$ Evaluación: 13 de octubre 2016

Aceptado: $\quad 15$ de octubre 2016 


\begin{abstract}
Manter um olhar crítico com relação aos programas de formação de professor é fundamental, tanto para pensar sobre sua pertinência, como para avançar no desenvolvimento destes programas frente a uma formação que aporte e que se desenvolva no marco da justiça social e equidade. No entanto, embora muitos programas de formação de professor defenda a justiça social, seus estudantes não são formados em um ambiente nesta perspectiva. Nos Estados Unidos, o debate atual ocorre em torno de qual é a melhor visão: se os programas que se baseiam em práticas de campo (1.0) ou aqueles que focam em aspectos técnicos da formação, assim como em obter resultados maiores em provas padronizadas (2.0). O certo é que nenhuma destas visões aviva em suas práticas valores imprescindíveis e inerentes a uma formação em justiça social, por isso torna-se explícita a necessidade de transformação dos programas de formação de professores.
\end{abstract}

Palavras-chave: Justiça social, Equidade, Pedagogia, Programas de formação de professores, Sensibilidade cultural.

Este artículo ha sido traducido por María Angélica Suavita Ramírez.

El artículo original se publicó en: Zeichner, K. (2016). Advancing social justice and democracy in teacher education: teacher preparation 1.0, 2.0, and 3.0. Kappa Delta Pi Record, 52(4), 150-155. doi:10.1080/00228958.2016.1223986

\title{
Introducción
}

En la actualidad, alrededor de dos terceras partes de los profesores en Estados Unidos se preparan en institutos y programas universitarios, mientras el otro tercio se prepara en los relativamente nuevos programas no-universitarios dirigidos por escuelas distritales, proveedores sin ánimo de lucro y proveedores con fines lucrativos, y por varias combinaciones de diferentes interesados (Consejo de Investigación Nacional, 2010). Independientemente del tipo de programa de preparación y quién lo dirija, la mayoría de los educadores del profesorado reclaman que sus programas se centren en aspectos de justicia social y equidad. Este artículo plantea la preocupación de en qué grado las prácticas de los formadores de profesorado reflejan sus reclamaciones en relación a los programas centrados en la justicia social y la equidad.

\section{Preparación del profesorado 1.0 y 2.0}

En la última década, los programas de formación del profesorado en institutos y universidades han sido objeto de un ataque creciente por parte de una variedad de críticos, incluyendo al ex Secretario de Educación de los Estados Unidos, Arne Duncan (Kellor, 2013). Las críticas a esos programas se centran en asuntos sobre su rigor intelectual y relevancia práctica (Wilson, 2014). Ha habido críticas a los programas de formación del profesorado en institutos y universidades a lo largo de la historia de la preparación del profesorado universitario (Fraser, 2007). Lo que es nuevo sobre las actuales, como Wilson (2014) puntualiza, es el hecho de que, con la ayuda de filántropos, comités de expertos y grupos de abogados, el Departamento de Educación de los Estados Unidos y políticos (Zeichner y Pena-Sandoval, 2015), esas críticas se han acoplado a la aparición de un nuevo conjunto de programas no universitarios y universitarios que están destinados a "interrumpir" el campo de la formación del profesorado y estimular la innovación. Muchos de esos programas (p. e., Schorr, 2012) 
son fast tracks ${ }^{l}$ en los que los profesores completan la mayor parte de sus requisitos de certificación como profesores oficiales, después de unas pocas semanas de formación previa, y muchos de ellos se centran muy estrictamente en los aspectos técnicos de la enseñanza y el aumento de las puntuaciones en pruebas estandarizadas de los alumnos e ignoran otros aspectos de la enseñanza como trabajo profesional, tales como el aprendizaje de los profesores sobre cómo ejercer su juicio en el aula y adaptar su enseñanza en respuesta a las necesidades cambiantes de sus estudiantes (Zeichner, 2014). Muchos de esos programas están conectados con redes de trabajo de escuelas autónomas y sirven como proveedores de profesores a esas redes (Stitlizen y West, 2014). Algunos como el Relay Graduate School of Education, que recientemente ha abierto su decimotercera ubicación en Baton Rouge, se están expandiendo muy rápidamente a nivel nacional y preparando más profesores cada año (Zeichner y Conklin, 2006).

Los nuevos programas que han sido traídos al campo por los empresarios sociales para estimular la innovación en la educación del profesorado han sido proclamados como programas 2.0 (Gastic, 2014) y, por implicación, los programas de institutos y universidades (educación de profesorado 1.0) han sido declarados obsoletos. Por ejemplo, Gastic (2014) predice:

La próxima década verá la proliferación de los modelos preparatorios del profesorado 2.0 según sean mejor conocidos y más ampliamente reconocidos los beneficios de su enfoque colectivo para la formación del profesorado.... (p. 105)

Aquellos programas que no puedan unirse a esta comunidad de enseñanza pronto revelarán su obsolescencia y se encontrarán luchando entre sí para justificar su existencia. La demanda se desplazará hacia programas más relevantes, asequibles y flexibles, donde los maestros estarán sujetos a altos estándares profesionales de conocimientos y habilidades bajo consideración de firmes instructores y orientadores, quienes están comprometidos con mejorar la eficacia del desempeño docente. (p. 109)

Mi contienda es que, a pesar de que la mayoría de los formadores de docentes en programas estatales 1.0 y 2.0 se centran en temas de justicia social y equidad en la preparación de profesores, muy pocos programas en ambas categorías han promulgado en sus prácticas alguno de los valores clave de la formación del profesorado en justicia social.

\section{La ausencia de formación del profesorado culturalmente sensible}

Dos ejemplos de cómo los programas de formación del profesorado 1.0 y 2.0 han fallado a la hora de actualizar en su educación de profesores que los valores que estos adoptan están relacionados con cuestiones de poder y conocimiento y con temas de sensibilidad cultural ${ }^{2}$. Primero, está muy claro en estudios sobre la experiencia de los candidatos a docente de color en programas de facultades y universidades predominantemente blancas que a menudo hay una falta de reconocimiento y sensibilidad en programas en relación con los atributos lingüísticos y culturales que los candidatos a profesor de color

\footnotetext{
${ }^{1}$ El término anglosajón fast track, cuya traducción literal es 'vía rápida', se utiliza para hacer referencia a programas que incluyen protocolos o características para una rápida finalización del mismo.

${ }^{2}$ Hay también otras maneras en las que muchos programas se han quedado cortos en las prácticas que predican, incluyendo su fallo en cumplir sus promesas para diversificar la composición racial y étnica de las cohortes de candidatos a profesor. Tanto con esta cuestión como con las discutidas aquí hay ejemplos prometedores de éxito en programas aislados, pero una falta general de realización en el campo en general (Sleeter, Neal y Kumashiro, 2015).
} 
traen consigo a su preparación para la docencia, y la preparación de profesores en esas instituciones frecuentemente ignora las necesidades de candidatos docentes de color y se centra en la preparación de profesores blancos para enseñar a estudiantes de color (Villegas y Davis, 2008).

Mientras los programas de formación de profesores de institutos y universidades, en parte debido a los estándares estatales y los requisitos nacionales de acreditación, incluyen ahora cursos en educación multicultural y prácticas clínicas en escuelas con alto impacto de pobreza, la investigación ha continuado mostrando un sesgo abrumador de blancura que enmarca los discursos y prácticas en muchos programas de institutos y universidades (Sleeter, 2001). Aunque hay excepciones a este problema en las instituciones y programas de formación de profesorado que no son predominantemente blancos (p. e., Irving y Fenwick, 2011; Skinner, Garreton y Schultz, 2011), la mayoría de programas 1.0 alientan a sus candidatos para profesor a enseñar de maneras culturalmente sensibles en sus clases escolares de primaria y secundaria, a pesar de que no enseñan en formas culturalmente sensibles a sí mismos.

Por lo que se sabe acerca de los programas 2.0 que comenzaron a surgir en los EEUU a principios de 1990 con el inicio de Teach for America, ellos también ignoran el capital cultural y lingüístico que sus candidatos a maestros aportan a sus programas, y enseñan un conjunto estándar de conocimientos y habilidades para todos en el programa con poco o nada de adaptación de los distintos recursos y necesidades de sus estudiantes (Kretchmar y Zeichner, en prensa). Varios de estos nuevos programas, como Relay, centran de manera muy estricta la enseñanza a los candidatos a profesor en un conjunto de habilidades de manejo del aula (p. e., Lemov, 2010) para implementar con fidelidad y ni siquiera abordan la cuestión de la enseñanza culturalmente sensible en las aulas de educación primaria y secundaria. Su teoría de la acción es que, si los profesores utilizan las técnicas de enseñanza y de gestión que se les enseñan, serán capaces de elevar los resultados de las pruebas de los estudiantes y la justicia social y la equidad serán alcanzadas. Por lo general, ni los resultados de la escolarización más allá de los resultados de las pruebas, ni los costes de centrarse obsesivamente en el aumento de los resultados de las pruebas, se discuten en la literatura del programa 2.0 (Kretchmar y Zeichner, en prensa).

La mayoría de las investigaciones sobre las experiencias de los estudiantes de color en programas de formación del profesorado, se basa en la experiencia en programas de institutos y universidades. La conclusión de que los programas 2.0 son culpables del mismo problema de "daltonismo"s en la pedagogía que los programas 1.0 se deduce de la ausencia total en el programa 2.0 de literatura de cualquier discusión sobre la sensibilidad cultural de la pedagogía en la formación del profesorado y de la dominancia de un discurso de "helperism", donde el énfasis es salvar a los estudiantes de sus comunidades rotas en lugar de reconocer y aprovechar los valores y los fondos de los conocimientos que existen en estas comunidades.

\footnotetext{
` El término del texto original, "colorblindness", sugiere una ceguera al color, un daltonismo; es un término sociológico para referirse al hecho de hacer caso omiso a características raciales y así tratar a los individuos lo más uniformemente posible, en un aparente intento por acabar con la discriminación, pero que es cuestionado por ser en sí mismo una forma de racismo. Se le llama también ceguera racial (race blindness).

${ }^{4}$ R. Helper (1829-1909) criticaba la esclavitud, basándose en criterios económicos. Su libro The Impending Crisis of the South: How to Meet it fue altamente criticado, tanto por los abolicionistas, como por los defensores de la esclavitud, para quienes helperism significaba una forma de fanatismo especialmente peligrosa.
} 


\section{Conocimiento y poder en programas de formación docente}

Un segundo aspecto que refleja la contradicción frecuente entre un compromiso expreso hacia la justicia social en la formación del profesorado y las realidades del programa de prácticas se trata de la cuestión sobre qué conocimiento es el que cuenta en la educación de los maestros. Uno esperaría que los formadores de docentes en programas que adoptan una misión de justicia social apuesten por un enfoque democrático hacia el problema de qué conocimiento cuenta en la educación de los maestros. En este enfoque, los maestros de las escuelas primarias y secundarias que sirven como centros de prácticas clínicas en los programas, los miembros de la comunidad que proporcionan servicios educativos y de otro tipo en las comunidades fuera de las escuelas y las familias que envían a sus hijos a las escuelas públicas locales estarían comprometidos en la colaboración equitativa con los programas de formación del profesorado y los programas trabajarían para incorporar los conocimientos en las escuelas y las comunidades en su trabajo con los futuros maestros.

Aquí, a pesar de la retórica omnipresente en la literatura sobre la formación del profesorado en institutos y universidades acerca de la importancia de las asociaciones auténticas y equitativas con las escuelas en la preparación de los maestros (Goodlad, 1998) y de la aparición de movimientos para establecer escuelas de desarrollo profesional y escuelas asociadas como lugares para la formación del profesorado, para la mayoría estas asociaciones han estado muy centradas en la universidad y han replicado las relaciones de poder-conocimiento que existían en las formas tradicionales de la formación del profesorado universitario (Duffy, 1994; Murrell, 1998; Zeichner, 2009).

Por otra parte, muchos de los programas 2.0 han construido una falsa dicotomía entre la teoría y la práctica y han glorificado, sin crítica, las prácticas de enseñanza que son una parte de sus marcos ya establecidos (p. e., Farr, 2010), mientras demonizan la teoría como irrelevante para la práctica diaria en las aulas. Esta glorificación sin críticas de la práctica dentro de los marcos particulares no es lo mismo que la valoración y el acceso, el conocimiento y la experiencia de las escuelas. Es un enfoque muy centrado en los programas que no los abre hacia el desafío y la crítica, ni compromete educadores K-12 $2^{5}$ en la toma de decisiones significativas acerca de los programas. Por otra parte, la marginación de lo que se conoce como "teoría" en muchos de estos programas estrecha la preparación de los maestros demasiado y minimiza la importancia del contexto y la cultura en el trabajo de los profesores. Esta falta de atención a la cultura y al contexto en la preparación de los maestros tendrá un impacto negativo en el trabajo de los profesores (Zeichner, 2009).

Además, a pesar de la importancia incuestionable de las relaciones entre profesores, escuelas, familias y comunidades en la educación de los estudiantes (Cuban 1969) y el llamado en la literatura por una mayor atención a la preparación de maestros para trabajar en maneras respetuosas y justas con las familias y comunidades de sus estudiantes y por programas para acceder al conocimiento y a la experiencia en las comunidades locales al servicio de este objetivo (Zeichner y Melnick, 1996), ha habido poca atención a estas cuestiones en ambos programas 1.0 y 2.0 (Zygmunt y Clark, 2016).

${ }^{5}$ K-12 es un término que se refiere a la suma de la educación primaria y secundaria. 
A pesar de aislados ejemplos para preparar a los maestros para involucrar a las familias en la educación de sus hijos (es decir, para conseguir que se escuche más lo que la escuela tiene que decirles), hay aún menos ejemplos de esfuerzos para comprometer o trabajar en solidaridad con las familias y las comunidades (Zeichner, Bowman, Guillen y Napolitan, 2016).

Continuar lanzando en paracaídas a maestros bien intencionados desde los programas de formación del profesorado universitarios y no universitarios hasta escuelas públicas que saben muy poco acerca de sus estudiantes, sus familias y comunidades y que no están comprometidas con la participación de las familias y las comunidades en la enseñanza continuará ampliando las brechas de oportunidades y aprendizaje que han persistido. Es irónico que tan poco de este trabajo continúe en los programas de formación del profesorado que atraviesan los EEUU cuando muchos de ellos han reclamado el manto de la justicia social como base para su trabajo.

\section{Practicando los valores y compromisos de la justicia social y la democracia en la formación del profesorado: preparación del profesor 3.0}

Desafortunadamente, los debates actuales sobre la formación del profesorado en los EEUU son en su mayoría sobre cuál es la mejor visión: la preparación del maestro 1.0, con un mayor enfoque en la práctica clínica, o la preparación de los maestros 2.0, con un enfoque casi exclusivamente sobre los maestros "en formación" para participar en un conjunto de enseñanza y prácticas de manejo de la clase que supuestamente aumentarán los resultados de los exámenes. Ambas, formación del profesorado 1.0 y 2.0, reclaman el manto de la formación del profesorado para la justicia social, aunque, en realidad, ninguno de los enfoques lo practica. Con el fin de llevar a la práctica los valores y compromisos que se promulgan en relación con la justicia social y la equidad, los programas de formación de docentes necesitan transformarse de manera que modelen la enseñanza culturalmente sensible que defienden para los candidatos a maestros y que interrumpe las jerarquías de poder-conocimiento que han marginado las voces y experiencia de los profesores y miembros de la comunidad en la preparación de los maestros.

La formación del profesorado 3.0 rechaza la opción que está siendo ahora proporcionada en los debates políticos actuales y ofrece un modelo que se basa en una nueva arquitectura más democrática, donde la responsabilidad de educar a los maestros es más equitativamente compartida por diferentes grupos de interés (es decir, las escuelas, las universidades, las comunidades locales), que colaboran de manera equitativa (Zeichner, Payne y Brayko, 2015). En algunos casos, los interesados adicionales, tales como los sindicatos de profesores locales y organizaciones no lucrativas, también están involucrados en ayudar a dar forma a un programa. En este enfoque, la epistemología del programa reúne los conocimientos y la experiencia de la universidad, las escuelas y la comunidad local de manera más democrática, y la atención se centra en el trabajo con y para las comunidades, en lugar de evitárselas a los estudiantes. Aquí, la formación del profesorado se convierte en una parte de un proyecto más amplio de desarrollo de la comunidad y los maestros ven su trabajo en las aulas como parte de una lucha más 
amplia por la justicia social a través de un proceso más democrático de deliberación y colaboración que atraviesa las estructuras institucionales.

Zeichner (2010) y Noel (2013) proporcionan ejemplos del trabajo actual en los programas de formación del profesorado de los EEUU, que muestran el movimiento hacia el tipo de epistemología democrática, que es un elemento crucial en la formación del profesorado 3.0. Algunos formadores de docentes también han abogado por el modelo de residencia del profesorado como una estructura para un programa, que es más inclusivo, de diferentes formas de experiencia, ubicados en universidades, escuelas y comunidades locales (p. e., Berry, Montgomery y Snyder, 2008). Aunque creo que la colaboración institucional transversal que se incluye en residencias de profesores ofrece el potencial para representar una postura 3.0 hacia la formación del profesorado, en la realidad, los programas de residencia de maestros varían en formas que incluyen el conocimiento y la experiencia de diferentes grupos de interés y sus compromisos ideológicos y políticos (Zeichner, 2014).

Por ejemplo, pocos de los programas de residencia de profesores existentes, genuinamente incluyen el conocimiento y la experiencia de los educadores de la comunidad local, líderes y familias en el plan de estudios o emplean a miembros de la comunidad como mentores de los candidatos a maestro. Además, algunos de los programas de residencia existentes que involucran la colaboración de las universidades y organizaciones locales sin ánimo de lucro y preparan a los maestros para un conjunto específico de colegios concertados en un distrito no colaboran con los distritos. Por último, algunos programas de residencia que, en la superficie implican colaboración de las universidades, solo las incluyen superficialmente (Zeichner, 2014). Necesitamos mirar más allá de los organigramas del modo en que se accede y se utilizan los conocimientos y la experiencia de los diferentes compañeros.

Una vez que los socios de la universidad, la escuela y la comunidad están unidos en modos de colaboración equitativa, la cuestión de la pedagogía en sensibilidad cultural de la formación del profesorado comienza a hacerse más visible en los programas y su presencia o ausencia en diferentes partes del programa se convierte en un tema de discusión (Zeichner, Bowman, Guillen y Napolitan, 2016).

Por último, es importante tener en cuenta que la formación del profesorado 3.0 no es particular a ninguna estructura específica del programa. Mientras que el modelo híbrido de residencia del profesorado ofrece la posibilidad de incorporar los elementos centrales del enfoque, también es posible que existan programas 1.0 y 2.0 para avanzar en esta dirección. Al final, son la sustancia y la calidad de los programas de formación del profesorado, en lugar de las etiquetas añadidas a los programas, los que hacen la mayor diferencia en la preparación de los maestros comunitarios culturalmente sensibles. Es hora de que, los formadores de docentes en todos los campos, admitan cómo los programas de formación del profesorado se han quedado cortos en la práctica, en el modelado de ideales y en los compromisos que ellos cuentan a sus profesores para utilizar en las escuelas.

\section{Referencias}

Cuban, L. (1969). Teacher and community. Harvard Educational Review, 39(2), 253272. doi:10.17763/haer.39.2.n7614t6x509xl3n1 
Duffy, G. (1994). Professional development schools and the disempowerment of teachers and professors. The Phi Delta Kappan, 75(8), 596-600.

Farr, S. (2010). Teaching as leadership. San Francisco, CA: Jossey-Bass.

Fraser, J. (2007). Preparing America's teachers: A history. Nueva York: Teachers College Press.

Gastic, B. (2014). Closing the opportunity gap: Preparing the next generation of effective teachers. En R. Hess y M. McShane (Eds.), Teacher quality 2.O. (p. 91). Cambridge, MA: Harvard Education Press.

Goodlad, J. (1998). Educational renewal: better teachers, better schools. San Francisco, CA: JosseyBass

Irvine, J. J. y Fenwick, L. T. (2011). Teachers and teaching for the new millennium: the role of HBCUs. The Journal of Negro Education, 80(3), 197-208.

Keller, B. (20 de octubre de 2013). An industry of mediocrity. New York Times. Recuperado de http://www.nytimes.com/2013/10/21/opinion/keller-an-industry-ofmediocrity.html?_r=0

Kretchmar, K. y Zeichner, K. (en prensa). Teacher education 3.0: A vision for teacher education to impact social transformation. Journal of Education for Teaching.

Lemov, D. (2010). To teach like a champion: 49 techniques that put students on the path to college. San Francisco, CA: Jossey-Bass.

Murrell, P. (1998). Like stone soup: The role of professional development schools in the renewal of urban schools. Washington DC: American Association of Colleges for Teacher Education.

Murrell, P. (2001). Community teacher. Nueva York: Teachers College Press.

National Research Council. (2010). Preparing teachers: building evidence for sound policy. Washington: National Academies Press.

Noel, J. (2013). (Ed). Moving teacher education into urban schools and communities: Prioritizing community strengths. Nueva York: Routledge.

Schorr, J. (2012). A revolution begins in teacher education. Stanford Social Innovation Review. Recuperado de http://www.ssireview.org/articles/entry/

Skinner, E. A., Garreton, M. T. y Schultz, B. D. (2011). Grow your own teachers: Grassroots change for teacher education. Teaching for social justice. Nueva York: Teachers College Press.

Sleeter, C. (2001). Preparing teachers for culturally diverse schools: Research and the overwhelming problem of whiteness. Journal of Teacher Education, 52(2), 94-106. doi:10.1177/0022487101052002002

Stitzlein, S. M. y West, C. K. (2014). New forms of teacher education: Connections to charter schools and their approaches. Democracy and Education, 22(2), 1-10. Recuperado de http:/ / democracyeducationjournal.org/cgi/viewcontent.cgi? article=1 146\&context=home

Villegas, A. M. y Davis, D. E. (2008). Preparing teachers of color to confront racial/ethnic disparities in educational outcomes. En M. Cochran-Smith, S. Feiman-Nemser y D. J. McIntyre (Eds.), Handbook of research on teacher education (pp. 583-605). Nueva York: Routledge.

Wilson, S. (2014). Innovation and the evolving system of U.S. teacher preparation. Theory into Practice, 53(3), 183-195. doi:10.1080/00405841.2014.916569

Zeichner, K. (2007). Professional development school partnerships in a culture of evidence and accountability. School-University Partnerships, 1(1), 9-17. 
Zeichner, K. (2009). Teacher education and the struggle for social justice. Nueva York: Routledge.

Zeichner, K. (2014). The struggle for the soul of teaching and teacher education. Journal of Education for Teaching, 4O(5), 551-568. doi:10.1080/02607476.2014.956544

Zeichner, K., Bowman, M., Guillen, L. y Napolitan, K. (2016). Engaging and working in solidarity with local communities in preparing of the teachers of their children. Journal of Teacher Education, 67(4), 277-290. doi:10.1177/0022487116660623

Zeichner, K. y Conklin, H. (2006). Beyond knowledge ventriloquism and echo chambers: Improving the quality the debate on teacher education. Teachers College Record, 119(4), 124.

Zeichner, K. y Melnick, S. (1996). The role of community field experiences in preparing teachers for cultural diversity. En. K. Zeichner, S. Melnick, y M. L. Gomez (Eds.), Currents of reform in preservice teacher education (pp. 176-196). Nueva York: Teachers College.

Zeichner, K., Payne, K. y Brayko, K. (2015). Democratizing teacher education. Journal of Teacher Education, 66(2), 122-135. doi:10.1177/0022847114560908

Zeichner, K. y Pena-Sandoval, C. (2015). Venture philanthropy and teacher education policy in the United States. Teachers College Record, 117(5), 1-44.

Zygmunt, E. y Clark, P. (2015). Transforming teacher education for social justice. Nueva York: Teachers College Press.

\section{Breve CV del autor}

\section{Kenneth Zeichner}

Profesor en la universidad de Washington en la que ha impartido cursos de posgrado sobre formación de profesorado, concernientes al debate en relación con políticas educativas. Además, en esta misma universidad se desempeñó como director de formación de profesorado en el periodo de 2009-2013. Recibió su Ph.D. en psicología educativa de la Universidad de Syracuse y desde entonces permaneció como docente de la Facultad de Educación de la Universidad de Wisconsin-Madison; allí fue decano asociado en Formación de profesorado y educación internacional, así como profesor en el departamento de currículo e instrucción, y dirigió una asociación de escuelas para el desarrollo profesional. Ha sido miembro de la Junta de Directores de la Asociación Americana de Colegios para la Formación de Maestros, copresidente del Panel de Investigación en Educación de Maestros de Asociación Americana de Investigación Educativa (AERA) y Vicepresidente de AERA (División K), así como también, miembro de la Academia Nacional de Comités de Educación sobre Formación Docente y Desarrollo Profesional Docente. Ha recibido significativos premios y distinciones, entre los que se encuentran Distinguished Research Award en 1990, y el Premio Margaret B. Lindsey por Contribuciones Distinguidas a la Investigación sobre la Formación de Maestros en 2002. El interés de Zeichner por la mejora de la educación, específicamente por la formación de maestros, surge de su propia experiencia en escuelas públicas, puesto que, a pesar de ser altamente evaluado en éstas, pudo evidenciar diferencias significativas en relación con lo que eran capaces de hacer otros niños que habían estudiado en escuelas más favorecidas. Kenneth Zeichner pone sobre la mesa el debate en relación con la desigualdad educativa, el enfoque de los programas de formación del profesorado, la autonomía del docente, la sensibilidad cultural y otros aspectos relativos a justicia social. Email: kenzeich@uw.edu 